\title{
Mixed Solid and Cystic Mass in an Infant
}

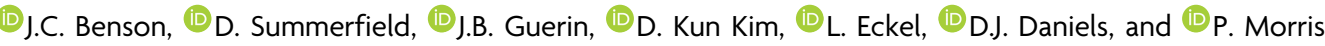

\section{ABSTRACT}

SUMMARY: Desmoplastic infantile tumors are rare supratentorial brain tumors that occur in pediatric patients. Desmoplastic infantile tumors are made up of 2 subtypes: desmoplastic infantile gangliogliomas and desmoplastic infantile astrocytomas. Desmoplastic infantile tumors are often identifiable on imaging on the basis of multiple characteristics. Nevertheless, pathologic analysis is required to confirm the diagnosis, particularly when the imaging features are atypical. Here, the radiology findings, surgical approach and subsequent management, and pathology of a desmoplastic infantile ganglioglioma are described.

ABBREVIATIONS: DIA = desmoplastic infantile astrocytoma; DIG = desmoplastic infantile ganglioglioma; DIT = desmoplastic infantile tumor

\section{Brief History}

The patient is a 3-month-old girl, born at 37 weeks' gestation after delivery was induced following the discovery of a 2-vessel umbilical cord. No intracranial or calvarial abnormalities had been reported on prenatal sonography. She presented with recentonset episodic lower left facial grimacing accompanied by decreased responsiveness. She also had progressive macrocephaly with a head circumference at the 96th percentile, severe frontal bossing, palpable splaying of the calvarial sutures, and a bulging anterior fontanelle.

\section{Imaging}

MR imaging showed a massive lesion in the right cerebral hemisphere with both cystic and solid components (Fig 1). The cystic aspects made up most of the mass and had a thin internal septation. The lobulated solid components were located centrally near the brain stem and were T2 isointense and heterogeneous with avid enhancement. Associated mass effect was present, including a leftward midline shift, right lateral ventricular effacement, and right basal ganglia and brain stem compression. Confluent T2 hyperintensity within the parenchyma posterior to the mass represented edema and/or gliosis. The cerebral aqueduct was compressed, causing left lateral and third ventricular dilation, though with minimal periventricular edema.

Received July 25, 2019; accepted after revision August 7.

From the Departments of Radiology (J.C.B., J.B.G., D.K.K., L.E., P.M.), Pathology (D.S.), and Neurosurgery (D.J.D.), Mayo Clinic, Rochester, Minesotta.

Please address correspondence to John C. Benson, MD, Mayo Clinic, Department of Radiology, 723 6th St SW, Rochester, MN 55902; e-mail: benson.john3@mayo.edu

http://dx.doi.org/10.3174/ajnr.A6226
The cystic component of the mass appeared to be extra-axial, evidenced by buckling and compression of the adjacent normal cortex. However, the solid components appeared to be at least partially intra-axial, with complete absence of normal temporal lobe parenchyma.

A massive, predominately cystic supratentorial mass presenting in an infant was suggestive of a desmoplastic infantile tumor (DIT), which includes both desmoplastic infantile gangliogliomas (DIGs) and astrocytomas (DIAs). However, mild-to-moderate restricted diffusion was seen within the solid components of the lesion, which would be atypical for this tumor (Fig 1). An infantile glioblastoma was also considered, given the shared imaging characteristics of both tumors and intralesional restricted diffusion. Other diagnostic considerations were thought to be less likely, including an embryonal tumor with multilayered rosettes, atypical teratoid/rhabdoid tumor, supratentorial ependymoma, and pleomorphic xanthoastryocytoma.

No metastases were present in the spine, and the patient underwent gross total resection of the tumor 2 days after presentation.

\section{Operative Report}

A near-gross total resection of the tumor was completed via a right craniotomy. The superficial cystic wall was thin and easily entered (Fig 2). The solid portion of the tumor was highly vascular and was firmly adherent to the adjacent parenchyma in several areas, particularly the right lateral brain stem. The tumor itself was firm and rubbery, consistent with a desmoplastic tumor, making the tumor difficult to aspirate and even challenging to cut. The right carotid artery and right middle cerebral artery were directly on the tumor and required careful separation. The tumor 


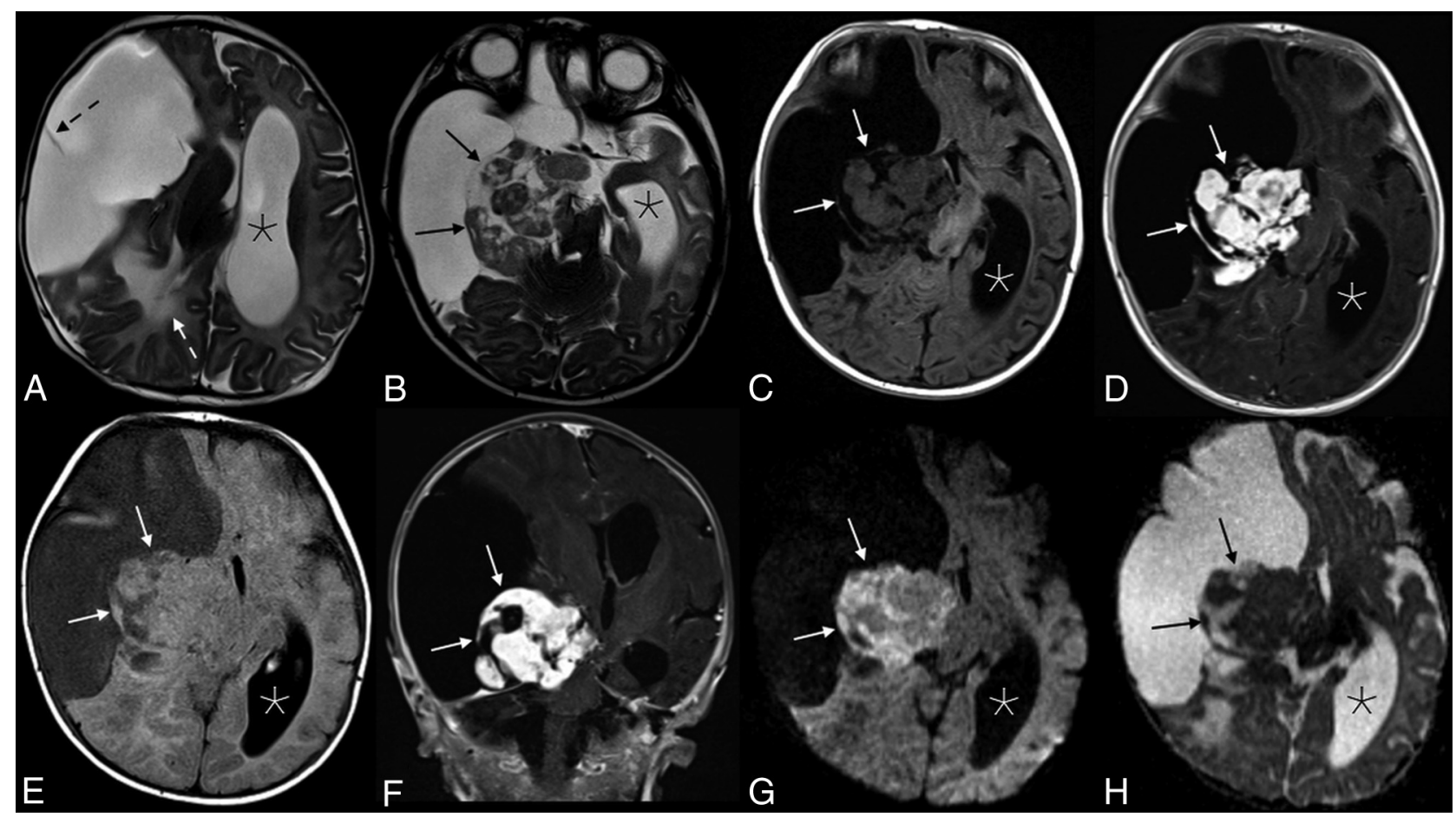

FIG 1. Appearance of the solid tumor component on various sequences. Axial T2 ( $A$ and $B$ ), axial T2 FLAIR (C), and T1 FLAIR (D) images showed a predominantly cystic mass in the right cerebral hemisphere with a heterogeneous solid component (black arrows in $B$, and $H$; white arrows in $C-G$ ) along the medial aspect of the tumor margin. A single thin septation was present within the cystic component (dashed black arrow, A). There is significant associated mass effect with T2 hyperintensity in the adjacent right parietal lobe white matter (white dashed arrow, A), a leftward midline shift, and compression of the right basal ganglia, brain stem, and right cerebral and middle cerebellar peduncles. The left lateral ventricle (asterisks) and third ventricle (not shown) were obstructed, causing marked ventricular dilation. Avid enhancement of the solid component is demonstrated in the axial $(D)$ and coronal $(E)$ TIWI + Gadolinium images. Heterogeneous mild intralesional restricted diffusion $(G$ and $H)$ is atypical for these tumors.

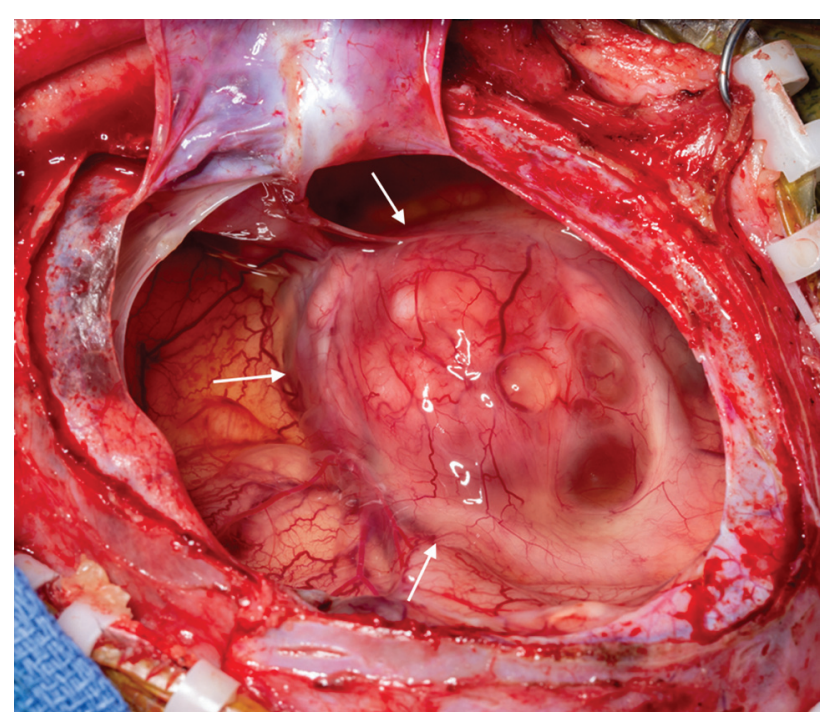

FIG 2. Intraoperative photograph of the tumor, after opening and draining of the cystic portion. The remaining mixed solid and cystic nodule is seen along the medial aspect of the tumor (arrows).

circumferentially surrounded the intersection of the right cranial nerve III and posterior cerebral artery, and a small amount of tumor was left at this location. The tumor came out piecemeal after careful dissection around these structures.

\section{Diagnosis: Desmoplastic Infantile Ganglioglioma}

Pathology. Histologically, DIGs and DIAs are considered histologic variants of a single World Health Organization grade I entity, with complete surgical resection resulting in long-term survival. ${ }^{1}$ Both have a prominent glial cell component characterized by desmoplasia with abundant extracellular matrix production and a fibroblast-like morphology (Fig 3). The distinction between DIG and DIA is made by the identification of a ganglion (neuronal) cell component, which is required for the diagnosis of the ganglioglioma entity. Methylation profiling studies of these tumors support both morphologies representing the same disease process, and long-term follow-up shows a similar prognosis. ${ }^{2}$ Some tumors show a minor component of poorly differentiated small round blue cells. However, this is not required for the diagnosis and was not present in this case. Finally, the proliferation rate of these tumors is typically low $(<2 \%){ }^{1}$

Photomicrographs of the tumor are presented in Fig 3, highlighting the desmoplastic component. The presence of rare synaptophysin-positive cells (ganglion cells) is sufficient for the diagnosis of DIG, and the glial fibrillary acidic protein (positive cells [glial cells]) is abundant. Tumor DNA was analyzed with a targeted 150 gene neuro-oncology next-generation sequencing panel (including BRAF, FGFR1, IDH1, IDH2, and NF1). Two variants of unknown significance were identified; however, no pathogenic mutations were identified. The Ki-67 labeling index, which is a marker of proliferative activity, was low in this tumor, 


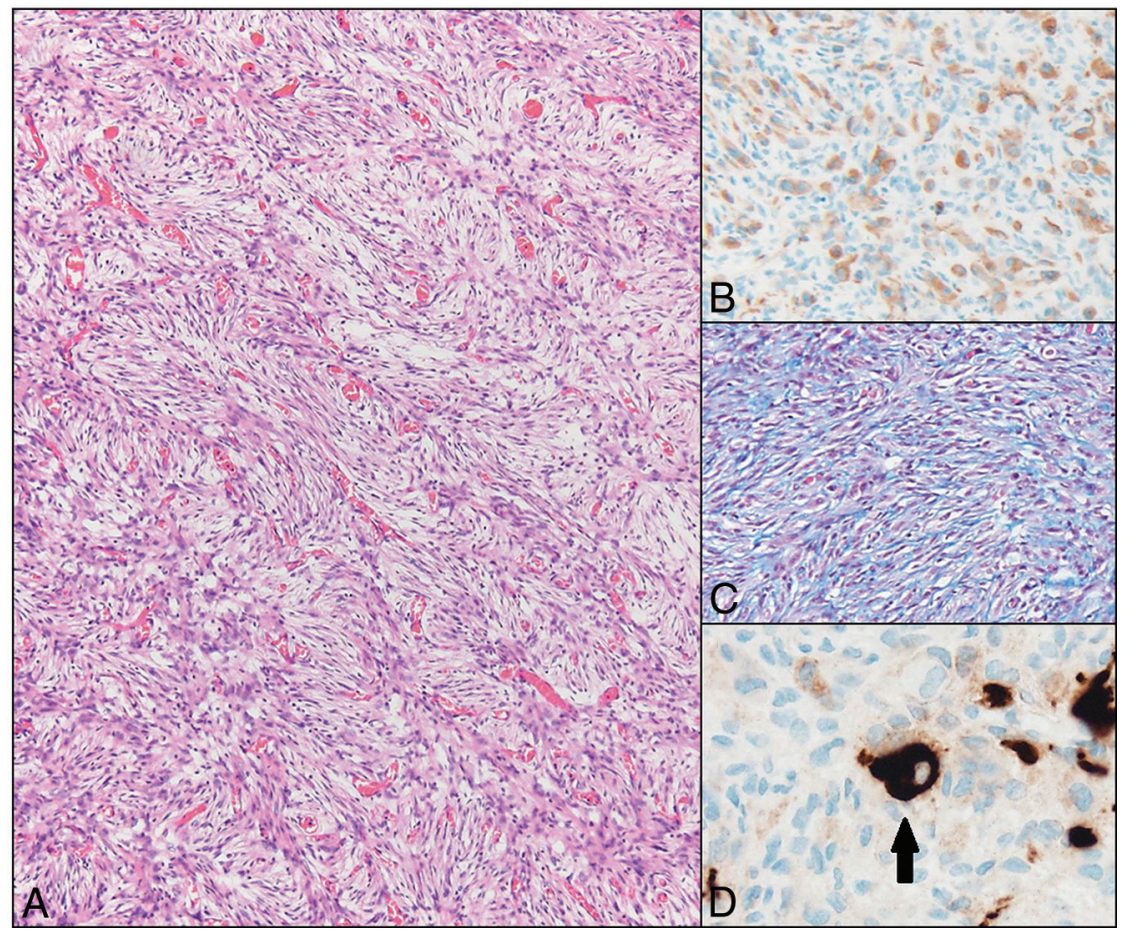

FIG 3. Hematoxylin eosin-stained photomicrograph demonstrates the characteristic fascicular/ storiform arrangement of the prominent spindle-shaped glial component of the tumor ( $A$, scaled from $100 \times$ ). These cells are glial fibrillary acid protein positive (confirming their glial origin) ( $B$, scaled from 200x), and an abundant pericellular connective tissue network is highlighted by the methyl blue component of a Mason trichrome stain (C, scaled from 100×). Rare ganglion cells are present (arrow), which are highlighted by Synaptophysin immunostaining ( $D$, scaled from $400 \times)$.
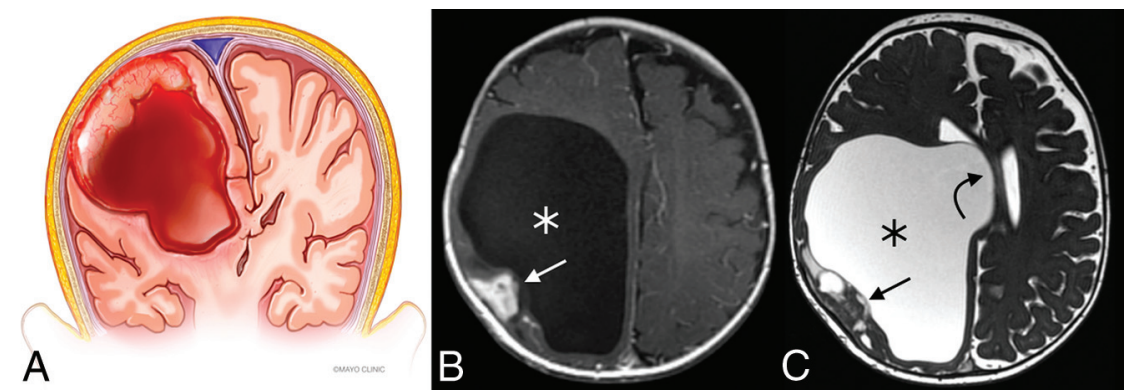

FIG 4. Illustration $(A)$ and example ( $B$ and $C$ ) of a DIT with typical features. The tumors are exclusively supratentorial and voluminous and are made up of both cystic (asterisks) components and a peripheral mural nodule (straight arrows). Significant associated mass effect and midline shift (curved arrow) are often present. Illustration (A), Used with permission of Mayo Foundation for Medical Education and Research. All rights reserved.

which supports the diagnosis of a DIG. BRAF mutations, which were absent in this tumor, are found in a minority of DITs. These histologic and molecular features are characteristic of a DIG.

\section{DISCUSSION}

DIGs are benign intra-axial CNS tumors. ${ }^{3,4}$ They are rare, accounting for $0.5 \%-1.0 \%$ of intracranial tumors. ${ }^{5,6}$ Nearly all DIGs occur in patients younger than 18 months of age with a median age at diagnosis of 5-6 months, though noninfantile cases have been reported. ${ }^{3,7-10}$ The male/female ratio is $1.7 .^{3}$ Most commonly, patients present with macrocephaly related to the size of the lesion and associated mass effect. Resulting seizures and other neurologic deficits may also occur. ${ }^{7}$ Despite the aggressive appearance of many DIGs on imaging, the prognosis of these tumors is often favorable, and surgical resection tends to be curative, though the voluminous size and high vascularity of these tumors can contribute to significant intraoperative blood loss. ${ }^{9-13}$ Although benign, extremely rare case reports do exist of DITs with malignant transformation as well as cerebrospinal metastases. $^{14,15}$

Multiple characteristic features make DITs recognizable entities on imaging, though the differentiation between DIG and DIA requires histologic analysis. The tumors are invariably supratentorial, contain solid and cystic components, and are often of substantial size. ${ }^{5,9}$ Involvement of multiple lobes is common, with a predilection for the frontoparietal regions. ${ }^{16,17}$ The solid component tends to be located peripherally, along the dural side of the tumor, and is usually T2 iso-/ hypointense to gray matter with avid enhancement (Fig 4). ${ }^{9,10}$ Typically, the dura or leptomeninges adjacent to the solid nodule or both demonstrate enhancement. ${ }^{9}$ Extremely rare purely solid and nonenhancing DIGs have been reported, as have DIGs in a suprasellar location. ${ }^{18}$

In the index case, the radiologic diagnosis was complicated by the presence of intralesional restricted diffusion. A review by Bader et $\mathrm{al}^{19}$ found no evidence of restricted diffusion in their 3 cases of DIT, and in only 1 of 32 DIT cases in a related review of the published literature. Infantile glioblastoma multiforme, conversely, had restricted diffusion in $2 / 2$ of the cases reviewed by Bader et al, and in 2/2 tumors in 18 prior publications reviewed by Bader et al. The mural nodule in our case was also located medial to the cystic component, which is a known, albeit rare, appearance of DIT. Nevertheless, the imaging characteristics of the presented case were overall highly suggestive of a DIT. The solid aspects of the mass demonstrated avid enhancement in our case, while infantile glioblastoma multiforme tends to enhance heterogeneously. ${ }^{19}$ 
Because of both the benignity of the tumor and the near-complete surgical resection, the neurosurgery team elected to proceed with a watch-and-wait approach. The patient had a right-sided cranial nerve III palsy, which was not unexpected because the residual tumor was near the third cranial nerve; this is expected to improve with time. The patient is scheduled to have follow-up imaging every 3 months for the first year; follow-up intervals will then be lengthened if the imaging findings are stable. If growth of the residual tumor is observed, the patient will likely need to undergo either a repeat resection or radiation therapy. An excellent long-term prognosis is expected.

\section{Case Summary}

- Imaging highly suggestive of a DIT (DIG/DIA): voluminous cystic mass with a peripheral mural nodule in an infant younger than 18 months of age

- Atypical features include central/medial location of solid component and intralesional restricted diffusion

- The main differential consideration is infantile glioblastoma multiforme, which tends to enhance heterogeneously

- Less likely diagnostic considerations: supratentorial ependymoma, embryonal tumor with multilayered rosettes, atypical teratoid/rhabdoid tumor, and pleomorphic xanthoastryocytoma

- Mass effect related to the tumor often requires urgent neurosurgical intervention; the surgical goal is to safely resect as much as possible and open CSF pathways.

Disclosures: Padraig Morris—UNRELATED: Royalties: textbook, Lippincott, Williams, \& Wilkins.

\section{REFERENCES}

1. Gessi M, Zur Muhlen A, Hammes J, et al. Genome-wide DNA copy number analysis of desmoplastic infantile astrocytoma and desmoplastic infantile gangliogliomas. J Neuropathol Exp Neurol 2013; 72:807-15 CrossRef Medline

2. Wang AC, Jones DTW, Abecassis IJ, et al. Desmoplastic infantile ganglioglioma/astrocytoma (DIG/DIA) are distinct entities with frequent BRAFV600 mutations. Mol Cancer Res 2018;16:1491-98 CrossRef Medline

3. Ho CY, Gener M, Bonnin J, et al. Diffusion, perfusion, and histopathologic characteristics of desmoplastic infantile ganglioglioma. $J$ Radiol Case Rep 2016;10:1-13 CrossRef Medline

4. Tamburrini G, Colosimo C, Giangaspero F, et al. Desmoplastic infantile ganglioglioma. Childs Nerv Syst 2003;19:292-97 CrossRef Medline
5. Tenreiro-Picon OR, Kamath SV, Knorr JR, et al. Desmoplastic infantile ganglioglioma: CT and MRI features. Pediatr Radiol 1995; 25:540-43 CrossRef Medline

6. Taranath A, Lam A, Wong C. Desmoplastic infantile ganglioglioma: a questionably benign tumour. Australas Radiol 2005;49: 433-37 CrossRef Medline

7. Romero-Rojas AE, Diaz-Perez JA, Lozano-Castillo A. Desmoplastic infantile ganglioglioma with late presentation. Neuroradiol J 2013; 26:649-54 CrossRef Medline

8. Gupta A, Karthigeyan M, Gupta K, et al. Atypical imaging in a desmoplastic non-infantile astrocytoma. Childs Nerv Syst 2017;33:51720 CrossRef Medline

9. Derinkuyu BE, Ucar M, Borcek AO, et al. Non-infantile variant of desmoplastic ganglioglioma: conventional and advanced MR imaging characteristics. Neuroradiol J 2015;28:259-63 CrossRef Medline

10. Gelabert-Gonzalez M, Serramito-García R, Arcos-Algaba A. Desmoplastic infantile and non-infantile ganglioglioma: review of the literature. Neurosurg Rev 2011;34:151-58 CrossRef Medline

11. Lababede O, Bardo D, Goske MJ, et al. Desmoplastic infantile ganglioglioma (DIG): cranial ultrasound findings. Pediatr Radiol 2001; 31:403-05 CrossRef Medline

12. Qaddoumi I, Ceppa EP, Mansour A, et al. Desmoplastic noninfantile ganglioglioma: report of a case. Pediatr Dev Pathol 2006;9:46267 CrossRef Medline

13. Sperner J, Gottschalk J, Neumann K, et al. Clinical, radiological and histological findings in desmoplastic infantile ganglioglioma. Child's Nerv Syst 1994;10:458-63 CrossRef Medline

14. Darwish B, Arbuckle S, Kellie S, et al. Desmoplastic infantile ganglioglioma/astrocytoma with cerebrospinal metastasis. J Clin Neurosci 2007;14:498-501 CrossRef Medline

15. Loh JK, Lieu AS, Chai CY, et al. Malignant transformation of a desmoplastic infantile ganglioglioma. Pediatr Neurol 2011;45:135-37 CrossRef Medline

16. VandenBerg SR. Desmoplastic infantile ganglioglioma and desmoplastic cerebral astrocytoma of infancy. Brain Pathol 1993;3:275-81 CrossRef Medline

17. Ng THK, Fung CF, Ma LT. The pathological spectrum of desmoplastic infantile gangliogliomas. Histopathology 1990;16:235-41 CrossRef Medline

18. Naylor RM, Wohl A, Raghunathan A, et al. Novel suprasellar location of desmoplastic infantile astrocytoma and ganglioglioma: a single institution's experience. J Neurosurg Pediatr 2018;22:397-403 CrossRef Medline

19. Bader A, Heran M, Dunham C, et al. Radiological features of infantile glioblastoma and desmoplastic infantile tumors: British Columbia's Children's Hospital experience. J Neurosurg Pediatr 2015;16:119-25 CrossRef Medline 